\title{
Identification of new progestogen- associated networks in mammalian ovulation using bioinformatics
}

\author{
Fang Yang ${ }^{1,2}$, Meng Wang ${ }^{1}$, Baoyun Zhang ${ }^{1}$, Wei Xiang ${ }^{1}$, Ke Zhang ${ }^{1}$, Mingxin $\mathrm{Chu}^{3}$ and Pingqing Wang ${ }^{1 *}$
}

\begin{abstract}
Background: Progesterone plays an essential role in mammalian ovulation. Although much is known about this process, the gene networks involved in ovulation have yet to be established. When analyze the mechanisms of ovulation, we often need to determine key genes or pathways to investigate the reproduction features. However, traditional experimental methods have a number of limitations.

Results: Data, in this study, were acquired from GSE41836 and GSE54584 which provided different samples. They were analyzed with the GEO2R and 546 differentially expressed genes were obtained from two data sets using bioinformatics (absolute $\log _{2} F C>1, P<0.05$ ). This study identified four genes (PGR, RELN, PDE10A and PLA2G4A) by protein-protein interaction networks and pathway analysis, and their functional enrichments were associated with ovulation. Then, the top 25 statistical pathway enrichments related to hCG treatment were analyzed. Furthermore, gene network analysis identified certain interconnected genes and pathways involved in progestogenic mechanisms, including progesterone-mediated oocyte maturation, the MAPK signaling pathway, the GnRH signaling pathway and focal adhesion, etc. Moreover, we explored the four target gene pathways. q-PCR analysis following hCG and RU486 treatments confirmed the certain novel progestogenic-associated genes (GNAl1, PRKCA, CAV1, EGFR, RHOA, ZYX, VCL, GRB2 and RAP1A).

Conclusions: The results suggested four key genes, nine predicted genes and eight pathways to be involved in progestogenic networks. These networks provide important regulatory genes and signaling pathways which are involved in ovulation. This study provides a fundamental basis for subsequent functional studies to investigate the regulation of mammalian ovulation.
\end{abstract}

Keywords: Progesterone, Bioinformatics, Progestogenic-associated, Mammalian ovulation

\section{Background}

Mammalian reproduction depends on the perfect ovulation of a fully differentiated oocyte capable of fertilisation. This process is triggered by hormonal release at the hypothalamic-pituitary-ovarian axis $[1,2]$. Ovulation is initiated by gonadotropin luteinising hormone (LH). This is a complex process that includes follicle-wall degradation, vasodilation, vascular dynamics and inflammation [2]. In this context, it is important to elucidate the mechanism of mammalian ovulation required for effective reproduction.

\footnotetext{
* Correspondence: wang_pq@21cn.com

'College of Bioengineering, Chongqing University, Chongqing 400030, China Full list of author information is available at the end of the article
}

Progesterone and its receptor (progesterone receptor, PGR) are both required in the ovulation process. The inhibition of progesterone synthesis by mifepristone is known to block ovulation in rats [2]. The anovulatory phenotype of the PGR-null mouse with a distinct defect in follicle-wall breakdown presents an opportunity to investigate molecular pathways. It is known that PGR controls the expression of a number of downstream target genes which regulate the ovulatory process [2-5]. Studies have identified certain progesterone-related factors including estradiol (E2), epidermal growth factor (Egf) [6], prostaglandin-endoperoxide synthase 2 (Ptgs2) [7], vascular endothelial growth factor (Vegfa) [8] and interleukin-6 (Il6) [9]. Furthermore, experiments using gene-null mice identified unruptured follicles and the 
failure of follicle-wall breakdown as the two causes of impaired ovulation. This analysis revealed novel gene signaling pathways which control the activity of PGR downstream to regulate ovulation, including PGR-HifAdamts1 (follicle-wall degradation), the PGR-Hif-Vegfa (vascular permeability) and PGR-Ppary-Il6-Ptgs2 (inflammation) pathways [2]. However, the process in which these systematic signaling networks are involved the regulation of ovulation has not been identified.

Unlike traditional methods, bioinformatics analysis can explore multi-component and multi-target molecular networks. In study of ovarian primordial follicle assembly, much of the information derived from sequencing data needs further analysis, including a systems biology approach to identify gene expression networks [10-15]. This approach can indicate novel factors and potential targets that correlate with ovarian disease. Grøndah et al. [16] described the transcriptome associated with oocyte development by in silico microarray analysis, identified differentially expressed genes by bioinformatics analysis, and further validated the identified genes through conducting quantitative real-time polymerase chain reactions (q-PCR) analysis and confirming the functional categories. These studies suggested that bioinformatics analysis based on sequencing data provided not only a systematic understanding of complex biological processes, but also a promising and efficient method to explore physiological functions in follicle development [13]. Current research focusing on the molecular pathways and networks involved in ovulation remains insufficient, and further bioinformatics analysis is needed.

The present study aims to explore gene networks and predict putative regulatory factors involved in ovulation by using bioinformatics analysis. Our objectives are to: 1) identify the most common and specific differentially expressed genes through investigating the proteinprotein interactions; 2) determine the key candidate genes by constructing biological gene networks involved in progesterone production during ovulation; 3) verify the candidate genes in progestogenic pathways. This study would improve the understanding of molecular functions by targeting specific pathways in the progestogenic networks, which might open new horizons into the investigation of the normal physiology of the ovaries.

\section{Methods}

\section{Data acquisition and analysis}

Data were acquired from the Gene Expression Omnibus (GEO) (http://www.ncbi.nlm.nih.gov/geo/) database. Two data sets, GSE54584 (this research clarified genetic alteration induced by the inhibition of cyclooxygenase or progesterone receptor) and GSE41836 (This study provided comprehensive information about regulated genes during late follicle development and ovulation induction), were contributed by Kenjiro et al. [17] and Cansu et al. [18], respectively. GSE41836 total contains 12 samples of cumulus oocyte complexes, among which only three samples with human Chorionic Gonadotropins (hCG) treatment were selected. GSE54584 total contains 18 samples, six samples including three samples with hCG treatment and three samples with RU486 (100 mg/ $\mathrm{kg})$ treatment in preovulatory were selected $[17,18]$. These selected samples were analyzed by the GEO2R (http://www.ncbi.nlm.nih.gov/geo/geo2r/) with respect to the control groups (samples with same dose normal saline treatment). Multiple testing was corrected using the Benjamini-Hochberg procedure to obtain the adjusted $P$-value $(P<0.05)$. Fold changes in the expression of individual genes were calculated and the genes with absolute $\log _{2} \mathrm{FC}>1$ were considered as differentially expressed genes (DEGs). Accordingly, DEGs determined from the selected data sets were identified as DEG41836 and DEG54584, the intersection of which was referred to as the overlapping DEGs.

\section{Protein-protein interaction (PPI) network analysis}

The Search Tool for the Retrieval of Interacting Genes (STRING, version 9.1) database (http://www.bork.emblheidelberg.de/STRING/) is a pre-computed global resource biological database that includes comprehensive known and predicted protein interaction information. In this study, the STRING 9.1 online tool was used to screen the overlapping DEGs with a confidence score over 0.4. The overlapping DEGs with hCG treatment were collected, then the relevant PPI network was constructed and visualized by Cytoscape software (version 3 . 0 ; http://cytoscape.org/) and was further analyzed by ClusterONE, a plugin of Cytoscape. Given that most of biological networks were scale-free, the hub genes were then selected with a connectivity more than 4 .

\section{Function and pathway enrichment analysis}

As a comprehensive set of functional annotation tools, the Database for Annotation, Visualization and Integrated Discovery (DAVID) has been used for the systematic and integrative analysis of large gene lists [19]. GO terms are significantly overrepresented in a set of genes from three aspects, including the cellular component, molecular function, and biological process $[13,20]$. In this study, the significant GO terms and Kyoto Encyclopaedia of Genes and Genomes (KEGG, http://www.genome.jp/) pathway enrichment analyses of the identified overlapping DEGs were performed using DAVID with the thresholds of $P$-value $<0.05$. Results were analyzed by using the IBM SPSS 19.0 software (International Business Machines Corporation, USA). 


\section{Network construction}

A biological network was generated by the progesteronemediated oocyte maturation pathway identified in KEGG. Based on preliminary relationships among differential genes, DEGs from the different treatment groups were assembled. The network analysis identified gene modules of the interconnected genes expressed co-ordinately in target pathways, followed by observation of genes that appeared to be involved in ovulation. The prediction of gene networks which were important to the ovulation process were validated using hCG- and RU486-stimulated experimental data (GSE54584). Details of the pathways, including constituent reactions, involved complexes, and network relationships, were elucidated in KEGG [20]. We recognized these pathways as elementary reactions that contained one reactant along with its resultant product to generate a gene network, regardless of small molecules.

\section{Q-PCR verification}

Sexually mature female rats (8-week-old, SpragueDawley) from Chongqing Medical University were used for the experimental verification. Rats were raised in controlled room conditions of constant temperature $\left(22-25{ }^{\circ} \mathrm{C}\right)$ and $12 \mathrm{~h}$ light/dark cycles with adlibitum access to food and water. All experiments were performed according to the guidelines of the National Institutes of Health, and the experimental protocol was approved by the animal-experiment committee.

Seleceted genes were validated through analysis. There were two treatment groups (6 Sprague-Dawley female rats per group for one experiment). The first group was intraperitoneally injected with Pregnant Mare Serum Gonadotropin (PMSG, $20 \mathrm{IU}$ ), and then $48 \mathrm{~h}$ later, hCG (20 IU) was administered through intraperitoneal injection. The second group was based on the procedures of the first group. Specifically, the second group of rats was gavaged with RU486 $(100 \mathrm{mg} / \mathrm{kg})$, a progesterone receptor antagonist, at $37 \mathrm{~h}$ post-PMSG. And then, hCG was administered through intraperitoneal injection. Rats were sacrificed and cumulus oocyte complex (COCs) samples were collected after hCG treatment $12 \mathrm{~h}$. Total RNA was acquired from ovary COCs using the extraction/isolation kit (BioTeke, Beijing, China). The RNA samples were reverse-transcribed into complementary DNA with the RevertAid First Strand cDNA Synthesis Kit (Takara, Dalian, China).

Each PCR reaction of $\mathrm{q}-\mathrm{PCR}$ consisted $2 \mu \mathrm{l}$ cDNA products, $7.5 \mu \mathrm{l}$ of the Power SYBR Green PCR Master Mix (Takara, Dalian, China), $4.5 \mu \mathrm{l}$ of water, and $1 \mu \mathrm{l}$ of gene-specific primers. q-PCR was performed in the following conditions: activation at $95{ }^{\circ} \mathrm{C}$ for $30 \mathrm{~s}$, followed by 40 cycles of $5 \mathrm{~s}$ at $95{ }^{\circ} \mathrm{C}$ and $30 \mathrm{~s}$ at $60{ }^{\circ} \mathrm{C}$. The final melting curve was added to examine the amplification quality, whereas expression of mRNA for $\beta$-actin was viewed as an internal control standard. The sequences of the primers from National Center for Biotechnology Information reference sequences are shown in Table 1 . The q-PCR results were analyzed using the Origin 8.0 software (Originlab Corporation, MA, US).

\section{Statistical analysis}

The statistical significance of change in the gene expression was determined using the Student's t-tests and posthoc Tukey's test. The data were shown as a fold change (mean \pm S.D) of values of at least three independent experiments and $P<0.05$ was considered statistically significant.

\section{Results}

\section{Functional and pathway analysis}

To identify the genes and pathways involved in progestogenic networks, two data sets (GSE41836 and GSE54584, hCG treatment samples) were subjected to intercommunity analysis using bioinformatics approaches. Total 546 common DEGs were obtained from the overlapping differential genes $\left(P<0.05\right.$, absolute $\log _{2}$ $\mathrm{FC}>1$ ), which were then used for PPI analysis and visualization (Additional file 1: Figure S1). Results from ClusterONE analysis identified four hub genes, including $P G R$, reelin (RELN), phosphodiesterase 10A (PDE10A) and phospholipase A2 group IVA (PLA2G4A) (Additional file 2: Table S1).

In order to further determine the key genes in progestogenic networks, functional and pathway enrichment analyses of these four candidate genes showed that PGR was involved in the 51 functions mainly related to ovulation, such as regulation of the ovulation cycle and steroid hormone receptor activity, and participated the signaling pathway of progesterone regulation follicle maturation. RELN was involved in 47 different functions, such as the activity of protein kinases, and PDE10A was mainly reported in the 20 functions related to the nucleotide binding domain, particularly playing a role in the purine metabolic signaling pathway. Finally, PLA2G4A participated cell proliferation regulation, metabolism and other aspects of the functions, and mainly related to the MAPK signaling pathway and the GnRH signaling pathway (Table 2) [21, 22].

Furthermore, we selected the prominent functions of four genes shown in Table 3, including the significant biological process, cellular component, and molecular function related to hCG treatment. In addition, the top 25 statistical pathways were also exhibited as the pathway enrichment analysis results of the 546 DEGs, considering the involved gene numbers and $P$-vaule (Fig. 1).

\section{Pathway regulation and gene network analysis}

Progestogenic pathways are a coordinated process initiated by hormones that activate ovulation. PGR and 
Table 1 Primer of prediction genes

\begin{tabular}{|c|c|c|c|}
\hline Gene name(symbol) & Primer sequences & Products size & Gene ID \\
\hline \multirow[t]{2}{*}{ Progesterone receptor $(P G R)$} & F:5'-GGTGGGCCTTCCTAACGAG-3' & $119 \mathrm{bp}$ & ID:5241 \\
\hline & R:5'-GACCACATCAGGCTCAATGCT-3' & & \\
\hline \multirow[t]{2}{*}{ Reelin (RELN) } & F:5'-CGCCTTTGGATTCGGGATCA-3' & $126 \mathrm{bp}$ & ID:24718 \\
\hline & R:5'-ATTCACACAGCCTGTGCCAG-3' & & \\
\hline \multirow[t]{2}{*}{ G protein subunit alpha i1 (GNA/1) } & F:5'-ACTTITCGTGCTTGCTGGGG-3' & $128 \mathrm{bp}$ & ID:25686 \\
\hline & R:5'-CCGGGATCTGTTGAAGCAGG-3' & & \\
\hline \multirow[t]{2}{*}{ Protein kinase $\mathrm{C}$, alpha (PRKCA) } & F:5'-CTGGGGAAGGGGAGTTTTGG-3' & $151 \mathrm{bp}$ & ID:24680 \\
\hline & R:5'-CTTCTCCACCATGGTGCACT-3' & & \\
\hline \multirow[t]{2}{*}{ Caveolin 1 (CAV1) } & F:'-CGCGCACACCAAGGAGATTG-3' & $143 \mathrm{bp}$ & ID:25404 \\
\hline & R:5'-TGCCGTCGAAACTGTGTGTC-3' & & \\
\hline \multirow[t]{2}{*}{ Epidermal growth factor receptor (EGFR) } & F:5'-ATCCTGATTGGTGCTGTGCG-3' & $114 \mathrm{bp}$ & ID:24329 \\
\hline & R:5'-CAACTGCTCGGATGGCTCTG-3' & & \\
\hline \multirow[t]{2}{*}{ Ras homolog family member $\mathrm{A}(\mathrm{RHOA})$} & F:5'-CAGCAAGGACCAGTTCCCAG-3' & $137 \mathrm{bp}$ & ID:117273 \\
\hline & R:5'-CGCAGGCGGTCATAATCTTC-3' & & \\
\hline \multirow[t]{2}{*}{ Zyxin $(Z Y X)$} & F:5'-TTCCAAGTTCAGCCCTGGTG-3' & $148 \mathrm{bp}$ & ID:114636 \\
\hline & R:5'-GGGCTTCTCCTTCTGCTGAG-3' & & \\
\hline \multirow[t]{2}{*}{ Vinculin (VCL) } & F:5'-AACAGCAGACCTGCCAAAGC-3' & $118 \mathrm{bp}$ & ID:305679 \\
\hline & R:5'-CAGCCACAAGTCCACGGATG-3' & & \\
\hline \multirow[t]{2}{*}{ Growth factor receptor bound protein (GRB) } & F:5'-AATCCCCAGAGCCAAGGCAG-3' & $145 \mathrm{bp}$ & ID: 84427 \\
\hline & R:5'-TCCAAACTTGACGGACAGGG-3' & & \\
\hline \multirow[t]{2}{*}{ RAS-relatedprotein-1a (RAPIA) } & F:5'-TGGGGAAGTCTGCTCTGACA-3' & $144 \mathrm{bp}$ & ID:295347 \\
\hline & R:5'-GGATCTCCAGCATGCACTGT-3' & & \\
\hline \multirow[t]{2}{*}{$\beta$-actin } & F:5'-AAAGACCTGTACGCCAACAC-3' & 137 bp & ID: 81822 \\
\hline & R:5'-GTCATACTCCTGCTTGCTGAT-3' & & \\
\hline
\end{tabular}

$F$ Forward, $R$ Reverse

RELN play an important role in this process. PGR is an upstream regulator of the progestogenic pathways in ovulation [23]. RELN acts as a factor promoting the end of follicle growth [24]. To verify whether the genes and networks of progestogenic pathways had potential theoretical significance, $P G R$ and $R E L N$ were analyzed to investigate the effects of differential expression in this process.

To characterize genes' alterations in preovulatory processes, gene expression data were analyzed related to hCG and RU486-treated experiments (GSE54584 data). Results demonstrated that significant changes in gene expression were observed comparing with the control groups. However, expression profiles of PGR in hCG and
RU486-treated groups were significantly different. This transcript is known to be involved in progesteronemediated oocyte maturation pathways and to interact with GNAI1, CPEB2 and KRAS (Fig. 2a). Four transcripts (MAPK1, CCNA2, IGF1 and BUB1) were significantly down-regulated in the two treatments (Fig. 2a and c). However, unlike the signaling pathways involving $P G R$, hCG and RU486-treated produced differential changes in $R E L N$ in follicular growth or focal adhesion pathways. Up-regulated transcripts in hCG-treated included PRKCA (>5-fold), CAV1 (>6-fold), EGFR (>3-fold), RHOA (> 2-fold), ZYX (> 2-fold), VCL (> 4-fold), RAP1A (>2-fold), and GRB2 (>2-fold). In contrast, these

Table 2 Information of ovulation candidate genes

\begin{tabular}{|c|c|c|c|c|}
\hline Name/symbol gene & Gene description & Functional description & Function number of gene & Pathway number of gene \\
\hline$\overline{P G R}$ & progesterone receptor & Process of ovulation & 51 & 1 \\
\hline RELN & reelin & factors binding & 47 & 2 \\
\hline PDE10A & phosphodiesterase $10 \mathrm{~A}$ & Process of metabolic & 20 & 1 \\
\hline PLA2G4A & $\begin{array}{l}\text { phospholipase A2, group IVA } \\
\text { (cytosolic, calcium-dependent) }\end{array}$ & positive regulation of factors activity & 11 & 2 \\
\hline
\end{tabular}


Table 3 The prominent functions enrichment analysis including the molecular function, biological process, and cellular component

\begin{tabular}{|c|c|c|}
\hline Term & $P$-Value & Percent \\
\hline \multicolumn{3}{|l|}{ Molecular function } \\
\hline Nucleotide binding & $1.83 \mathrm{E}-09$ & $14.41 \%$ \\
\hline Purine nucleotide binding & $5.26 \mathrm{E}-08$ & $12.33 \%$ \\
\hline Purine ribonucleotide binding & $5.64 \mathrm{E}-07$ & $11.67 \%$ \\
\hline Ribonucleotide binding & $5.64 \mathrm{E}-07$ & $11.67 \%$ \\
\hline Adenyl nucleotide binding & $6.34 \mathrm{E}-07$ & $10.17 \%$ \\
\hline Nucleoside binding & $1.19 \mathrm{E}-06$ & $10.24 \%$ \\
\hline Purine nucleoside binding & $1.33 \mathrm{E}-06$ & $10.17 \%$ \\
\hline Ion binding & $1.52 \mathrm{E}-06$ & $23.23 \%$ \\
\hline Cation binding & $2.29 \mathrm{E}-06$ & $22.92 \%$ \\
\hline Metal ion binding & 4.40E-06 & $22.64 \%$ \\
\hline Adenyl ribonucleotide binding & $6.25 \mathrm{E}-06$ & $9.51 \%$ \\
\hline Magnesium ion binding & 7.90E-04 & $2.95 \%$ \\
\hline Guanyl ribonucleotide binding & 0.002959769 & $2.57 \%$ \\
\hline Guanyl nucleotide binding & 0.002959769 & $0.31 \%$ \\
\hline AMP binding & 0.005264347 & $0.66 \%$ \\
\hline \multicolumn{3}{|l|}{ Biological process } \\
\hline Multicellular organism reproduction & 0.0001 & $3.13 \%$ \\
\hline Reproductive process in a multicellular organism & 0.0001 & $3.13 \%$ \\
\hline Morphogenesis of a branching structure & 0.0001 & $1.25 \%$ \\
\hline Tissue morphogenesis & 0.0001 & $2.01 \%$ \\
\hline Epithelium development & 0.0001 & $2.22 \%$ \\
\hline Tube morphogenesis & 0.0001 & $1.56 \%$ \\
\hline Development of primary sexual characteristics & 0.0001 & $1.08 \%$ \\
\hline Sexual reproduction & 0.0001 & $2.92 \%$ \\
\hline Sex differentiation & 0.0002 & $1.25 \%$ \\
\hline Gamete generation & 0.0004 & $2.50 \%$ \\
\hline Branching morphogenesis of a tube & 0.0014 & $0.90 \%$ \\
\hline Ovulation cycle process & 0.0021 & $0.56 \%$ \\
\hline Ovulation cycle & 0.0026 & $0.56 \%$ \\
\hline Female pregnancy & 0.0075 & $0.66 \%$ \\
\hline Steroid hormone receptor signaling pathway & 0.0251 & $0.28 \%$ \\
\hline Phospholipid metabolic process & 0.0472 & $1.15 \%$ \\
\hline Phosphorus metabolic process & 0.000018103 & $5.94 \%$ \\
\hline \multicolumn{3}{|l|}{ Cellular component } \\
\hline Vesicle & 0.023864658 & $3.16 \%$ \\
\hline Cytoplasmic vesicle & 0.026121829 & $3.09 \%$ \\
\hline Extracellular matrix & $2.72 \mathrm{E}-06$ & $2.60 \%$ \\
\hline Extracellular region part & $7.54 \mathrm{E}-06$ & $5.35 \%$ \\
\hline Proteinaceous extracellular matrix & $9.05 \mathrm{E}-06$ & $2.47 \%$ \\
\hline Cell projection & 7.94E-06 & $3.82 \%$ \\
\hline Extracellular space & 0.008741592 & $3.23 \%$ \\
\hline Neuron projection & 0.011946122 & $1.70 \%$ \\
\hline
\end{tabular}




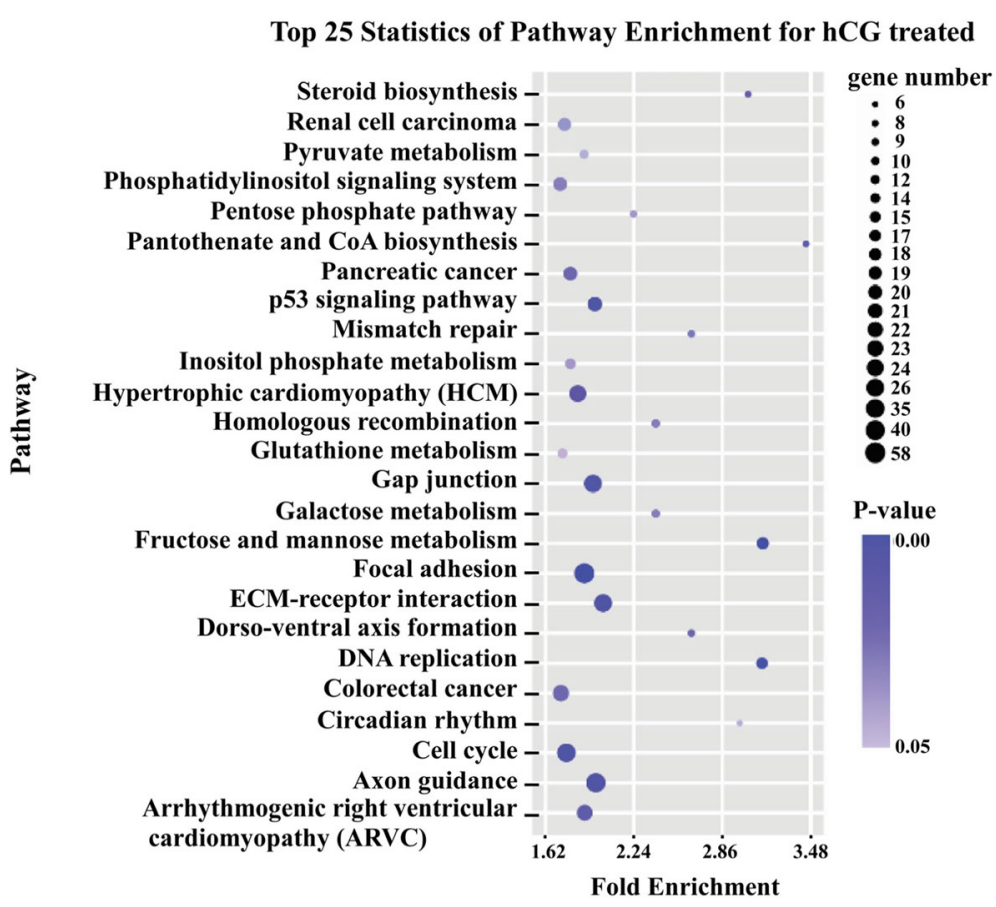

Fig. 1 Pathway enrichment analysis. The top 25 statistics pathways identified by pathway enrichment analysis of the 546 DEGs, considering the involved gene numbers and $P$-vaule

transcripts were down-regulated in RU486-treated (Fig. 2b, d and e). In addition, some genes (MAPK1, BUB1, CANA2 and IGF1) involved in the RELN pathway were down-regulated in both hCG and RU486 treatment (Additional file 3: Table S2). These expression differences were considered relevant to the induction of ruptured and un-ruptured follicles and other features such as inflammation, tissue remodeling, extracellular matrix release, and steroid metabolism $[2,7,17]$.

New gene network construction of the progestogenic pathway was based on primary network relationships between the four genes and pathways of PGR and RELN. The network combined 8 pathways (progesterone-mediated oocyte maturation, MAPK signaling pathway, GnRH signaling pathway, purine metabolism, PI3K-Akt signaling pathway, Wnt signaling pathway, ECMreceptor interaction, and focal adhesion) with node genes PIK3R5, MAPK, and ADCY1, then added nine predicted genes (GNAI1, PRKCA, RHOA, ZYX, VCL, RAP1A, CAV1, EGFR and GRB2) to the network. A combined method was taken, regardless of small molecules or undetected genes (Fig. 3).

\section{Validation of predicted results by $\mathrm{q}-\mathrm{PCR}$}

In order to verify whether the differential genes (GNAI1, PRKCA, RHOA, ZYX, VCL, RAP1A, CAV1, EGFR and $G R B 2)$ in the progesterone signaling pathway were indeed regulated by PGR, they were detected by q-PCR. This was the quantitative detection of samples from
hCG- and RU486-treated rats after the extraction of COCs. The results of $\mathrm{q}-\mathrm{PCR}$ showed that the expression of PGR was almost completely inhibited after RU486 treatment, and the expression of RELN was also decreased (Fig. 4a and b). In addition, it was found that the nine predicted genes (GNAI1, PRKCA, RHOA, ZYX, $V C L, R A P 1 A, C A V 1, E G F R$ and GRB2) exhibited a differential change (Fig. 4c-k). The expression of $V C L$, RHOA, RAP1A and GRB2 decreased by about 3-fold compared with the control group, and the significant decreases of ZYX, GNAI1, CAV1 and PRKCA were about 2-fold. The change of EGFR was inhibited, although the trend became below par. This may also be subject to the regulation of other factors in the process of ovulation.

\section{Discussion}

One of the challenges in mammalian ovulation research is to investigate the basic regulatory and physiological mechanisms [25]. The present study has identified genes with special importance in unique developmental processes. Selectively GSE41836 and GSE54584 data served as accessible data sets for studying the progestogenicrelated gene networks in mammals [10]. Herein, we highlighted ovarian physiology and identified many new progestogenic-related genes in this study.

This study also identified several novel initiation factors of ovulation. Regulations of hormones and steroidogenesis are regarded as an initiation process, which provides detailed fundamental information on the 


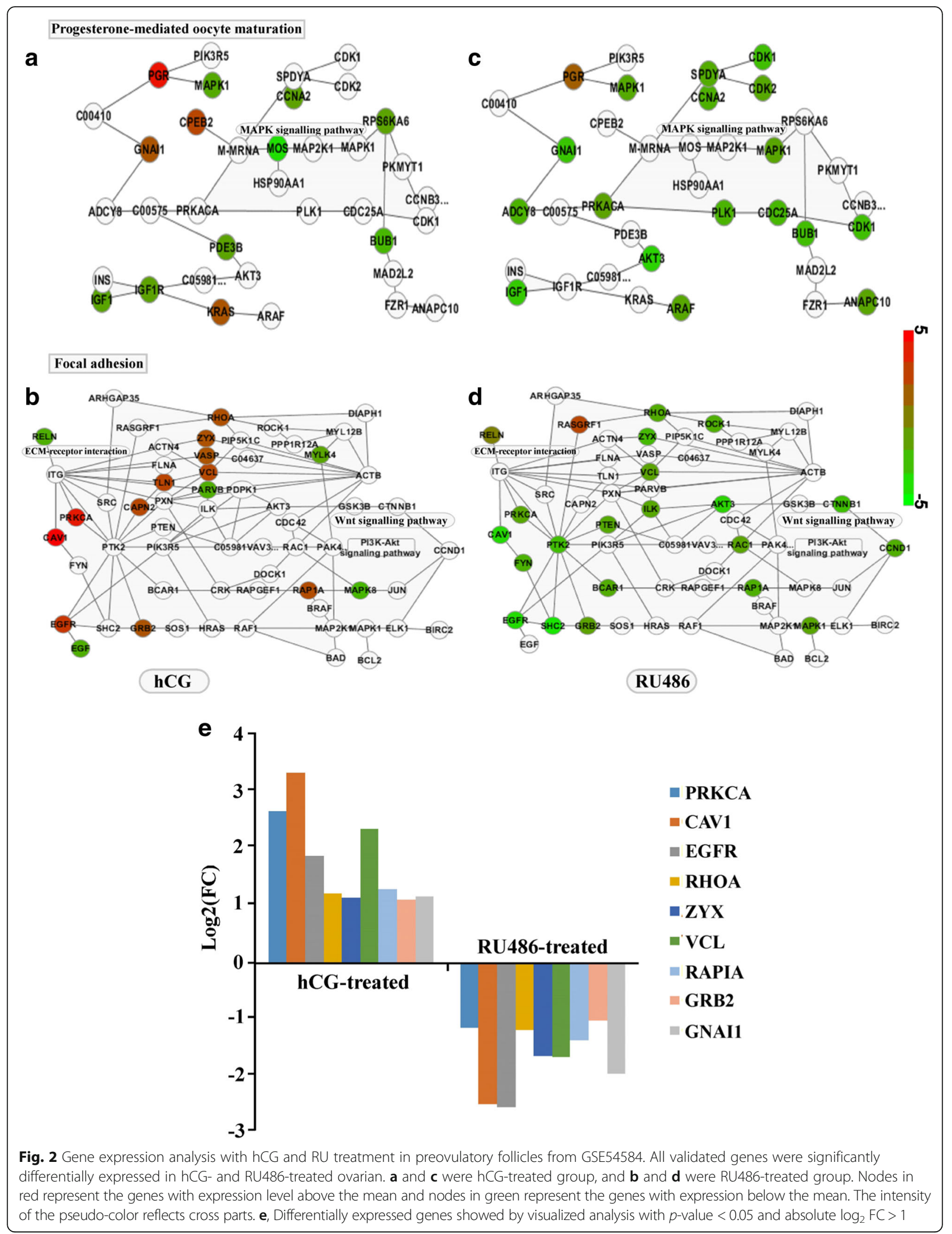




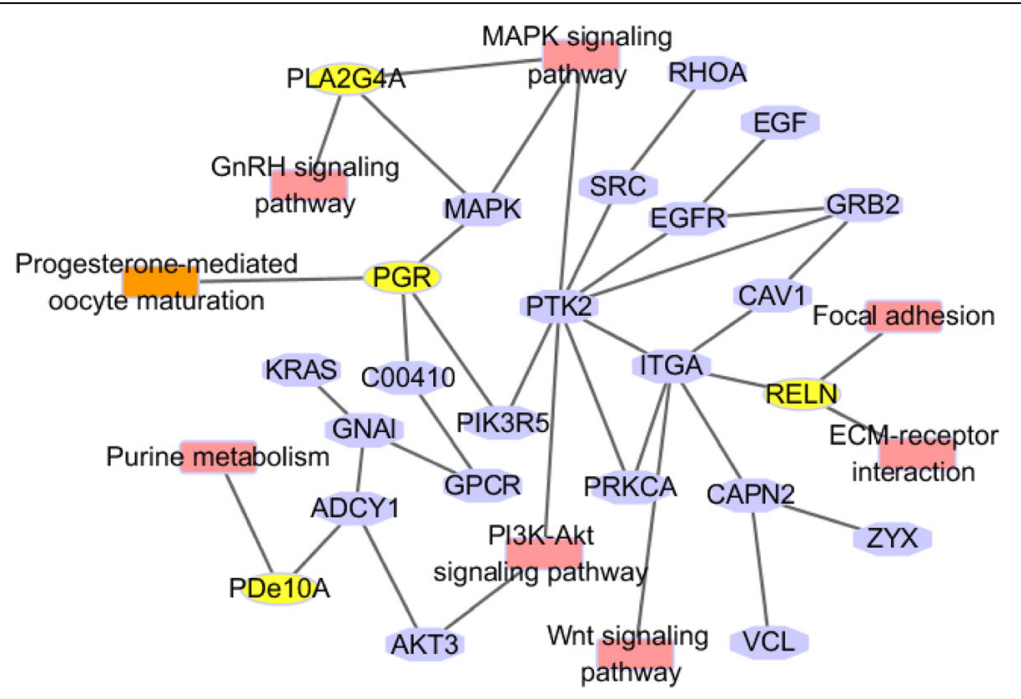

Fig. 3 Visualized progesterone-related genes network. Nodes in different colors represent different target genes or pathways. Purple sexangles represent node genes (MAPK, SRC, PTK2, ITGA, CAPN2, GPCR, ADCY1) and predicted target genes (GNAI1, PRKCA, CAV1, EGFR, RHOA, ZYX, VCL, GRB2 and RAP1A) in this study; yellow color ellipses represent genes between networks; and red color rectangles reflect pathways involved in progestogenic networks

profound changes regulated by gene networks in ovulation. Hormone (FSH and LH) induced primary follicle maturation and eventually approached the pre-ovulatory stage in mice and rats [2, 26]. Pituitary-released LH forms the LH surge which subsequently initiates the estrous cycle in rodents in response to the high levels of estrogen [27]. The steroid hormone progesterone, regulated by the LH surge, plays a key role in ovulation [28]. The effects of progesterone were mediated by PGR, and its expression was observed in the granulosa cells of preovulatory follicles following hCG treatment in mice and rats [29]. PGR was accompanied by the coordinated expression of progesterone biosynthetic enzymes, including steroidogenic acute regulatory protein (Star) and cholesterol side-chain cleavage enzyme (Cyp11a1) [30, 31]. An expression increase of Star and Cyp11a1 was observed following hCG stimulation, but a decrease was in RU486treated mice, which revealed a pattern of expression related to the progestogenic pathway.

In the progestogenic pathway, PGR expression is induced primarily in granulosa cells of the pre-ovulatory follicles [2]. PGR is a transcription factor involved in multiple processes including oocyte maturation, ovulation, ovulation cycle process, epithelial cell development, and steroid hormone receptor signaling pathways. PGR directly mediates ovulation and indirectly regulates other ovulation-related factors. The expression of RELN, a regulator of follicle growth factor like IGF2 [24], decreased in hCG-treated rats (Fig. 2c). It binded with the receptor LRP8 to regulate follicular growth in cattle. MAPK8IP1 encoding LRP8 intracellular interacting partner was also expressed in granulosa cells of the dominant follicle. This differential expression pattern suggested an RELN/LRP8/MAPK8IP1 paracrine interaction to regulate follicular growth until ovulation [32]. PDE10A, one of the PDE families, is also expressed in bovine cumulus cells by cAMP-PDE activity [33]. Mammalian oocytes initiate meiosis in the foetus but remain arrested at the diplotene stage of the first meiotic prophase, before being potentiality to resume by the ovulatory luteinising hormone pulse [34]. High levels of cAMP keep oocytes in the meiotic arrest phase by activating cAMP-dependent protein kinase. As for $P D E 10 A$, effects of ovulation on the process are uncertain. Functional and pathway analysis found that PLA2G4A was involved in the MAPK and GnRH signaling pathways. Many studies have confirmed that ovulation is associated with an LH/hCG-dependent induction of PLA2G4A in granulosa cells via the adenylyl cyclase/cAMP pathway [35]. Kurusu et al. [36] found that PLA2G4A optimizes ovulation and fertilization when coupled with prostaglandin endoperoxide synthase in female mice lacking PLA2G4A. This study found that four genes (PGR, RELN, PDE10A, and PLA2G4A) developed an expression pattern consistent with a progestogenic pathway during ovulation in mammals. This analysis suggests that these four genes can form a network of cellular pathways or nodes to regulate ovulation.

Progestogenic pathway plays an important role in the "story" of follicle maturation and ovulation. In order to explore extensions of the progestogenic pathway, this study investigated the four genes that were involved in this pathway. An analysis of the four genes from the KEGG pathway and PPI showed that MAPK, PIK3R5, $P T K 2, I T G A$, and $A D C Y 1$ had the most regulatory connections to adjacent genes (Fig. 4). These genes are 

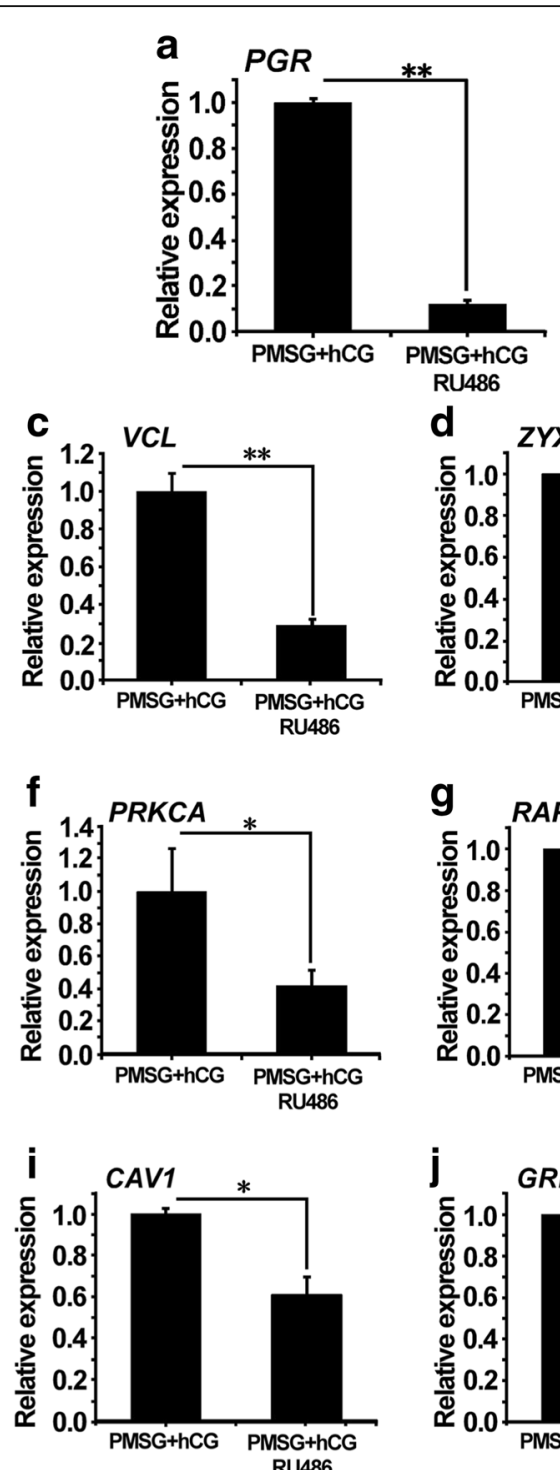
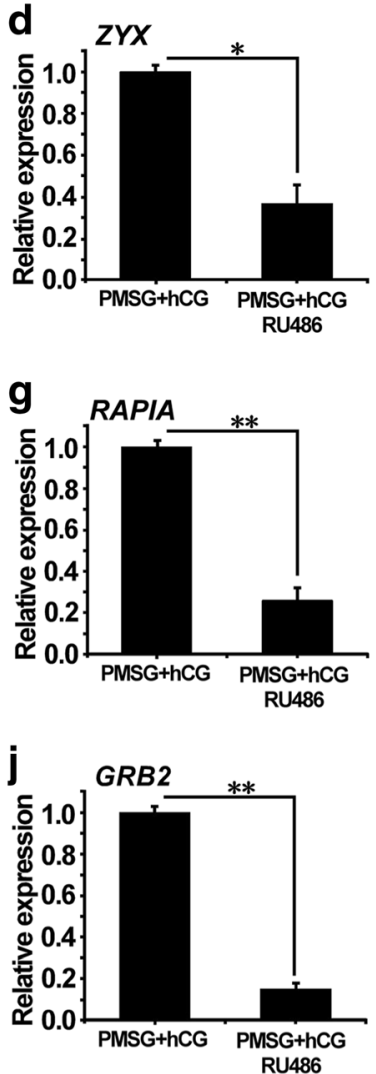
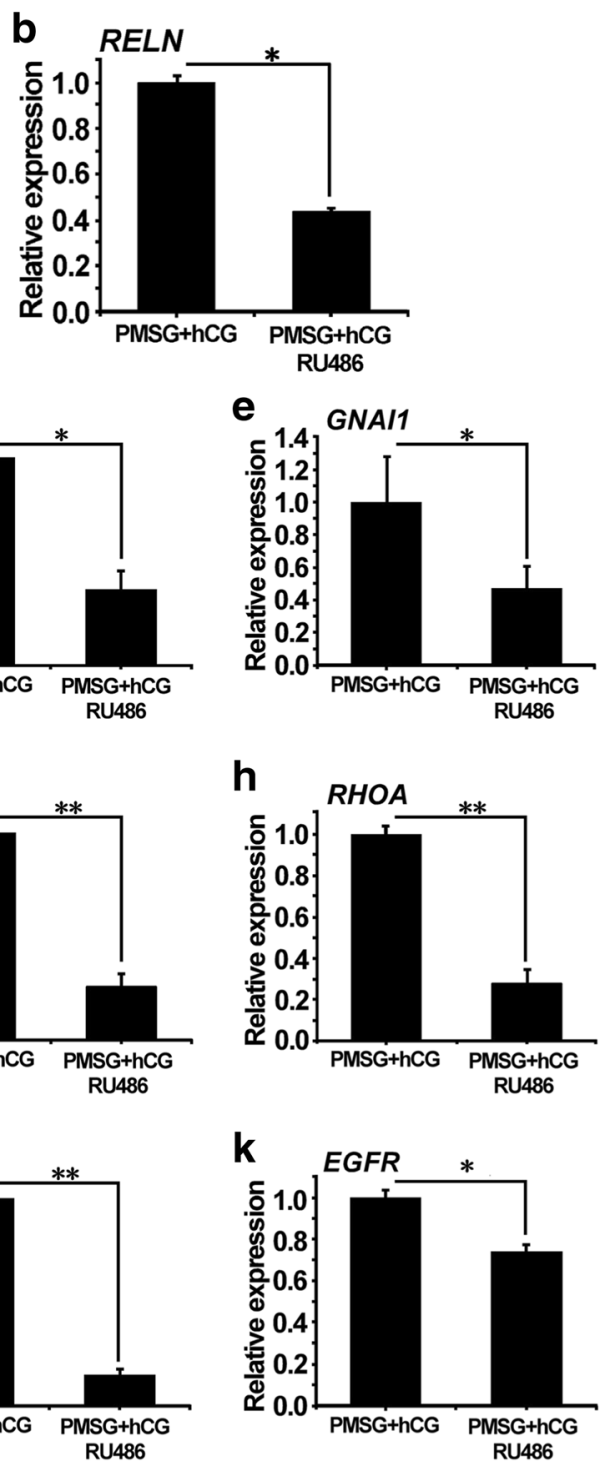

Fig. 4 Validation of progesterone-related predicted genes by q-PCR analysis. The two groups of rats were treated by PMSG and hCG or PMSG, hCG and RU486. Total RNA of COCs was extracted for q-PCR analysis of the genes expression. $\mathbf{a}-\mathbf{b}$ : $P G R$ and RELN expression; $\mathbf{c}-\mathbf{k}$ : expression of the nine genes (GNAI1, PRKCA, CAV1, EGFR, RHOA, ZYX, VCL, GRB2 and RAP1A, respectively). Different asterisks indicate statistically significant differences $\left({ }^{*}, P<0.05 ;{ }^{* *}, P<0.01\right)$. Error bars represent S.D.

considered important in regulating features involved in the progestogenic network or as downstream factors of the network. Genes involved in each pathway may act in concert with accomplishing distinct processes during ovulation including prostaglandin synthesis (Ptgs 2 and PLA2G4A) [7], cholesterol uptake (Ldlr and Scarb1) [37], regulation of progesterone synthesis (Star) [30], inactivation of estrogen (Sult1e1) [38], and downstream effectors of LH signaling (Pgr, Cebpb, Areg, Ereg, and Adamts1) [6, 26, 29, 39]. These processes regulate inflammation, follicle-wall degradation, hormone balance and the induction of certain paracrine factors. In addition, genes encode proteins involved in various biology process, including reproductive developmental processes and granulosa cell expansion ( $T g f b 2$, Foxl2, Pde3a, Esr2, Fshr, Ccnd2) [40], amino acid degradation and ketogenesis (Hmgcs2, Cpt1b) [41]. These results presented candidate genes involved in independent cellular pathways to carry out relevant functions in progestogenic pathways.

Interestingly, when the rats were subjected to hCG and RU486 treatment, the results indicated that most of the genes involved in PGR and RELN pathways were differentially regulated. Furthermore, these results illustrated that focal adhesion, EMC-receptor interaction and MAPK signaling pathways were affected in hCG- 
and RU486-treated rats. MAPK signaling pathways provoke ovulation and collapse of the follicle, which were stimulated by a variety of exotic signals (MAP kinases 1 and 3, extracellular-regulated protein kinases 1 and 2 (ERK1/2)) [42]; the Wnt-signaling and PI3K-Akt signaling pathways were not significantly affected by hCG treatment (Fig. 2c). Conti and Makker [43, 44] found that the PI3K/PTEN/Akt signaling pathway and cAMP/ ERK/EGF network induced oocyte and meiotic maturation. It is noteworthy that the PDE family participated in the cAMP pathway and research suggests that it also regulates ovulation. Sasseville et al. [33] indicated the establishment of a model of cell-specific expression of PDEs in the ovarian follicle. This suggested that Pde3a was a major isoform of oocyte PDE, Pde4d was the major granulosa cell PDE isoforms and PDE10A was expressed in cumulus cells. Cell-specific expression of cAMP-PDEs in ovarian follicles has implications for understanding the hormonal regulation of folliculogenesis [45]. Further studies are needed to determine how Pde10a-Adcy1 participates in progestogenic pathways. Pathway analysis and gene validation indicated that RELN could participate in PI3K-Akt signaling by PTEN, but the molecular mechanism of this process remained unclear. However, when RELN combines with receptor LRP8 to regulate follicular growth, the pattern of LRP8 expression correlates with the capacity of follicles to synthesize higher steroid levels in cattle [32]. Subsequently, the phase of follicular development, including the LH surge, changes the relationship between progestin and androgen. Enzymes required for progestin include hydroxy-delta-5-steroid dehydrogenase, 3 betaand steroid delta-isomerase (Hsd3b2), and Cyp11a1. This suggests that RELN and LRP8 may be regulated in progestogenic pathways, but human studies have not verified this regulation. Pathway validation indicated the PRKCA exhibited significant changes in RELN regulation (Fig. $2 \mathrm{~b}$ and d). This validation also connected Ptk2 to progesterone-mediated oocyte maturation which confirmed the relationship between RELN and PGR. Details of this interaction require further investigation. Relative expression results using q-PCR also found that GNAI1 was considered as a PGR indirect regulatory factor, but this process was not previously associated with ovulation. GNAI1, PRKCA, RHOA, ZYX, VCL, RAP1A, CAV1, $E G F R$ and GRB2 produced changes in progestogenic pathways and these factors were considered as ovulation-associated factors. PLA2G4A is involved in the progestogenic pathway by paracrine regulation, which plays an important role in ovulation. The ovulatory surge of LH regulates the expression of each component of the $\mathrm{PGE}_{2}$ synthesis-metabolism-transport pathway to mediate key ovulatory events including cumulus expansion, rupture and release of oocytes [7].

\section{Conclusions}

In sum, a systematic bioinformatics analysis approach could provide an overview of a particular developmental process. Results suggested that the four genes ( $P G R$, RELN, PDE10A and PLA2G4A) were involved in novel progestogenic networks (Fig. 3). These networks provided important regulatory genes and signaling pathways which were involved in ovulation. At the same time, hCG- and RU486-treated experiments confirmed that candidate genes (GNAI1, PRKCA, RHOA, ZYX, VCL, RAP1A, CAV1, $E G F R$ and GRB2) were involved in ovulation.

This study has shown that the above genes are selectively or highly differentially expressed in progestogenic pathways, which provides important information for future downstream analysis to identify the significant genes in ovulation and develop effective strategies for fertility control.

\section{Additional files}

Additional file 1: Figure S1. The significant modules in the protein protein interaction network with MCODE. Nodes denoted proteins (genes). In specific, the yellow denoted seed proteins; the red denoted the up-regulated proteins; and the green denoted down-regulated proteins; the width of edges was determined according to the combined score of the protein-protein interaction relationships. Abbreviation: MCODE, Molecular Complex Detection. (DOCX $115 \mathrm{~kb}$ )

Additional file 2: Table S1. Four genes information from analysis of Cluster One. (DOCX $13 \mathrm{~kb}$ )

Additional file 3: Table S2. The list of significantly differentially expressed genes of the pathways involving RELN and PGR. (DOCX $14 \mathrm{~kb}$ )

\section{Abbreviations}

Cyp11a1: Cholesterol side-chain cleavage enzyme; E2: Estradiol; ECG: Equine chorionic gonadotrophin; FSH: Follicle-stimulating hormone;

GnRH: Gonadotropin-releasing hormone; GO: Gene Ontology; HCG: Human chorionic gonadotrophin; Hsd3b2: Hydroxy-delta-5-steroid dehydrogenase, 3 beta- and steroid delta-isomerase; II6: Interleukin 6; LH: Luteinizing hormone; OCC: Oocyte cumulus complex; PDE10A: Phosphodiesterase 10A;

PGR: Progesterone receptor; PLA2G4A: Phospholipase A2 group IVA; Ptgs2: Prostaglandin-endoperoxide synthase 2; RELN: Reelin; Vegfa: Vascular endothelial growth factor

\section{Acknowledgments}

We gratefully thank Dr. Muhammad Farrukh Nisar, Dr. Feng Jianguo and Dr. Kong Xiangjun for grammar revision.

\section{Funding}

This work was supported by National Natural Science Foundation of China (Grant No. 31372287), National Biological Breeding Capacity Building and Industrialization Projects (2014-2573), Ministry of Agriculture transgenic major projects of China (2014ZX0800952B), and the Agricultural Science and Technology Innovation Program of China (ASTIP-IAS13).

\section{Availability of data and materials}

The data supporting the results of this article are included and cited within the article and its additional files.

\section{Authors' contributions}

FY carried out the molecular genetic studies, completed in the sequence alignment and drafted the manuscript. MW helped to finish the sequence analysis. FY and PW designed this study and performed the statistical analysis. BZ, 
MC, WX and KZ participated in its design and coordination, and provided valuable advice. All authors read and approved the final manuscript.

\section{Ethics approval and consent to participate}

This study does not contain any studies with human participants or animals performed by any of the authors. Informed consent was obtained from all individual participants included in the study.

\section{Consent for publication}

Not applicable.

\section{Competing interests}

The authors declare that they have no competing interests.

\section{Publisher's Note}

Springer Nature remains neutral with regard to jurisdictional claims in published maps and institutional affiliations.

\section{Author details}

${ }^{1}$ College of Bioengineering, Chongqing University, Chongqing 400030, China. ${ }^{2}$ Medical Molecular Biology Research Center, School of Basic Medical Sciences, Southwest Medical University, Luzhou, Sichuan 646000, China. ${ }^{3}$ Key Laboratory of Farm Animal Genetic Resources and Germplasm Innovation of Ministry of Agriculture, Institute of Animal Science, Chinese Academy of Agricultural Sciences, Beijing 100193, China.

Received: 4 May 2017 Accepted: 27 March 2018

Published online: 03 April 2018

\section{References}

1. Deady LD, Sun JA. Follicle rupture assay reveals an essential role for follicular adrenergic signaling in drosophila ovulation. PLoS Genet. 2015; 11(10):e1005604.

2. Kim J, Bagchi IC, Bagchi MK. Control of ovulation in mice by progesterone receptor-regulated gene network. Mol Hum Reprod. 2009;15(12):821-8.

3. Kadariya I, Wang J, ur Rehman Z, Ali H, Riaz H, He J, Bhattarai D, Liu JJ, Zhang SJ. RNAi-mediated knockdown of inhibin a subunit increased apoptosis in granulosa cells and decreased fertility in mice. J Steroid Biochem Mol Biol. 2015;152:161-70.

4. Cho J, Kim H, Kang DW, Yanagisawa M, Ko C. Endothelin B receptor is not required but necessary for finite regulation of ovulation. Life Sci. 2012 91(13-14):613-7.

5. Burns KH, Yan C, Kumar TR, Matzuk MM. Analysis of ovarian gene expression in follicle-stimulating hormone beta knockout mice. Endocrinology. 2001; 142(7):2742-51.

6. Blaha M, Nemcova L, Kepkova KV, Vodicka P, Prochazka R. Gene expression analysis of pig cumulus-oocyte complexes stimulated in vitro with follicle stimulating hormone or epidermal growth factor-like peptides. Reporod Biol Endocrinol. 2015;13:113.

7. Duffy DM. Novel contraceptive targets to inhibit ovulation: the prostaglandin E2 pathway. Hum Reprod Update. 2015;21(5):652-70.

8. Mauro A, Martelli A, Berardinelli P, Russo V, Bernabò N, Di Giacinto O, Mattioli M, Barboni B. Effect of antiprogesterone RU486 on VEGF expression and blood vessel remodeling on ovarian follicles beforeovulation. PLoS One. 2014:9(4):e95910.

9. Molyneaux KA, Schaible K, Wylie C. GP130, the shared receptor for the LIF/ IL6 cytokine family in the mouse, is not required for early germ cell differentiation, but is required cell-autonomously in oocytes for ovulation. Development. 2003:130(18):4287-94.

10. Fan HY, Liu Z, Shimada M, Sterneck E, Johnson PF, Hedrick SM, Richards JS MAPK3/1 (ERK1/2) in ovarian granulosa cells are essential for female fertility. Science. 2009;324(5929):938-41.

11. Zhang JY, Wu Y, Zhao S, Liu ZX, Zeng SM, Zhang GX. Lysosomes are involved in induction of steroidogenic acute regulatory protein (StAR) gene expression andprogesterone synthesis through low-density lipoprotein in cultured bovine granulosa cells. Theriogenology. 2015;84(5):811-7.

12. Zhou C, Teng WJ, Zhuang J, Liu HL, Tang SF, Cao XJ, Qin BN, Wang CC, Sun CG. Analysis of the gene-protein interaction network in glioma. Genet Mol Res. 2015;14(4):14196-206.

13. Wang $Y$, Huang $L$, Wu $S$, Jia $Y$, Yang $Y$, Luo L, Bi A, Fang $M$. Bioinformatics analyses of the role of vascular endothelial growth factor in patients with non-small cell lung Cancer. PLoS One. 2015; 10(9):e0139285.

14. Nilsson E, Zhang B, Skinner MK. Gene bionetworks that regulate ovarian primordial follicle assembly. BMC Genomics. 2013;14:496.

15. Li C, Zhu B, Chen J, Huang X. Novel prognostic genes of diffuse large B-cell lymphoma revealed by survival analysis of gene expression data. Onco Targets Ther. 2015;8:3407-13.

16. Grøndahl ML, Borup R, Vikeså J, Ernst E, Andersen CY, Lykke-Hartmann K. The dormant and the fully competent oocyte: comparing the transcriptome of human oocytes from primordial follicles and in metaphase II. Mol Hum Reprod. 2013;19(9):600-17.

17. Tsubota K, Kanki M, Noto T, Nakatsuji S, Oishi Y, Matsumoto M, Nakayama H. Altered gene expression profile in ovarian follicle in rats treated with indomethacin and RU486. J Toxicol Sci. 2015;40(3):413-25.

18. Agca C, Yakan A, Agca Y. Estrus synchronization and ovarian hyperstimulation treatments have negligible effects on cumulus oocytecomplex gene expression whereas induction of ovulation causes major expression changes. Mol Reprod Dev. 2013;80(2):102-17.

19. Kutmon M, Evelo CT, Coort SLA. Network biology workflow to study transcriptomics data of the diabetic liver. BMC Genomics. 2014;15:971.

20. Kou Y, Zhang S, Chen X, Hu S. Gene expression profile analysis of colorectal cancer to investigate potential mechanisms using bioinformatics. Onco Targets Ther. 2015:8:745-52.

21. Wismann J, Willoughby D. Gender differences in carbohydrate metabolism and carbohydrate loading. J Int Soc Sports Nutr. 2006;3:28-34.

22. Zhang W, Zhou T. A sparse reconstruction approach for identifying gene regulatory networks using steady-state experiment data. PLoS One. 2015; 10(7):e0130979.

23. Wissing ML, Kristensen SG, Andersen CY, Mikkelsen AL, Høst T, Borup R, Grøndahl ML. Identification of new ovulation-related genes in humans by comparing the transcriptome of granulosa cells before and after ovulation triggering in the same controlled ovarian stimulation cycle. Hum Reprod. 2014;29(5):997-1010

24. Nivet AL, Vigneault C, Blondin P, Sirard MA. Changes in granulosa cells' gene expression associated with increased oocyte competence in bovine. Reproduction. 2013;145(6):555-65.

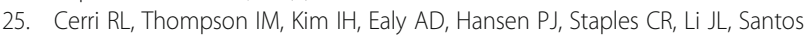
JE, Thatcher WW. Effects of lactation and pregnancy on gene expression of endometrium of Holstein cows at day 17 of the estrous cycle or pregnancy. J Dairy Sci. 2012;95(10):5657-75.

26. Bylander A, Gunnarsson L, Shao R, Billig H, Larsson DG. Progesteronemediated effects on gene expression and oocyte-cumulus complex transport in the mouse fallopiantube. Reprod Biol Endocrinol. 2015;13:40.

27. Breen SM, Andric N, Ping T, Xie F, Offermans S, Gossen JA, Ascoli M. Ovulation involves the luteinizing hormone-dependent activation of $\mathrm{Gq} / 11$ in granulosa cells. Mol Endocrinlo. 2013;27(9):1483-91.

28. Tam KK, Russell DL, Peet DJ, Bracken CP, Rodgers RJ, Thompson JG, Kind KL Hormonally regulated follicle differentiation and luteinization in the mouse is associated with hypoxia inducible factor activity. Mol Cell Endocrinol. 2010;327(1-2):47-55.

29. Robker RL, Russell DL, Espey LL, Lydon JP, Malley BW, Richards JS. Progesterone-regulated genes in the ovulation process: ADAMTS-1 and cathepsin L proteases. Proc Natl Acad Sci U S A. 2000:97(9):4689-94.

30. Kowalewski MP, Gram A, Boos A. The role of hypoxia and HIF1a in the regulation of STAR-mediated steroidogenesis in granulosa cells. Mol Cell Endocrinol. 2015;401:35-44.

31. Cui J, Shen Y, Li R. Estrogen synthesis and signaling pathways during ageing: from periphery to brain. Trends Mol Med. 2013;19(3):197-209.

32. Fayad T, Lefebvre R, Nimpf J, Silversides DW, Lussier JG. Low-density lipoprotein receptor-related protein 8 (LRP8) is upregulated in granulosa cells of bovine dominantfollicle: molecular characterization and spatiotemporal expression studies. Biol Reprod. 2007:76(6):466-75.

33. Sasseville M, Albuz FK, Côté N, Guillemette C, Gilchrist RB, Richard FJ. Characterization of novel phosphodiesterases in the bovine ovarian follicle. Biol Reprod. 2009;81(2):415-25

34. Thomas RE, Thompson JG, Armstrong DT, Gilchrist RB. Effect of specific phosphodiesterase isoenzyme inhibitors during in vitro maturation of bovine oocytes on meiotic and developmental capacity. Biol Reprod. 2004; 71(4):1142-9.

35. Diouf MN, Sayasith K, Lefebvre R, Silversides DW Sirois J, Lussier JG Expression of phospholipase A2 group IVA (PLA2G4A) is upregulated by 
human chorionic gonadotropin in bovine granulosa cells of ovulatory follicles. Biol Reprod. 2006;74(6):1096-103.

36. Kurusu S, Sapirstein A, Bonventre JV. Group IVA phospholipase $A_{2}$ optimizes ovulation and fertilization in rodents through induction of and metaboliccoupling with prostaglandin endoperoxide synthase 2. FASEB J. 2012;26:3800-10.

37. Park JY, Jang H, Curry TE, Sakamoto A, Jo M. Prostate androgen-regulated mucin-like protein 1: a novel regulator of progesterone metabolism. Mol Endocrinol. 2013;27(11):1871-86.

38. Gerrits $H$, Paradé $M C$, Koonen-Reemst AM, Bakker NE, Timmer-Hellings $L$, Sollewijn Gelpke MD, Gossen JA. Reversible infertility in a liver receptor homologue-1 (LRH-1)-knockdown mouse model. Reprod Fertil. 2014; 26(2):293-306

39. Fan HY, Liu Z, Johnson PF, Richards JS. CCAAT/enhancer-binding proteins(C/ EBP)- $\alpha$ and $-\beta$ are essential for ovulation, luteinization, and the expression of key target genes. Mol Endocrinol. 2011;25:253-68.

40. Regan SL, McFarlane JR, O'Shea T, Andronicos N, Arfuso F, Dharmarajan A, Almahbobi G. Flow cytometric analysis of FSHR, BMRR1B, LHR and apoptosis in granulosa cells and ovulation rate in merino sheep. Reproduction. 2015; 150(2):151-63.

41. Dunning KR, Cashman K, Russell DL, Thompson JG, Norman RJ, Robker RL. Beta-oxidation is essential for mouse oocyte developmental competence and early embryo development. Biol Reprod. 2010;83:909-18.

42. Duggavathi R, Murphy BD. Development. Ovulation signals. Science. 2009; 324(5929):890-1.

43. Conti M, Hsieh M, Zamah AM, Oh JS. Novel signaling mechanisms in the ovary during oocyte maturation and ovulation. Mol Cell Endocrinol. 2012; 356(1-2):65-73.

44. Makker A, Goel MM, Mahdi AA. PI3K/PTEN/Akt and TSC/mTOR signaling pathways, ovarian dysfunction, and infertility: an update. J Mol Endocrinol. 2014;53:R103-18.

45. Campos-Chillon F, Farmerie TA, Bouma GJ, Clay CM, Carnevale EM. Effects of aging on gene expression and mitochondrial DNA in the equine oocyte and follicle cells. Reprod Fertil Dev. 2015;27(6):925-33.

\section{Submit your next manuscript to BioMed Central and we will help you at every step:}

- We accept pre-submission inquiries

- Our selector tool helps you to find the most relevant journal

- We provide round the clock customer support

- Convenient online submission

- Thorough peer review

- Inclusion in PubMed and all major indexing services

- Maximum visibility for your research

Submit your manuscript at www.biomedcentral.com/submit

) Biomed Central 